ANTIMICROBIALS

\title{
Time to Fight the New with the Old-Old Antibiotics in New Role
}

\author{
Anuradha Vinod ${ }^{1}$, Bhaskar Shenoy ${ }^{2}$
}

\begin{abstract}
Current medical practice is seeing a rise of multidrug-resistant bacteria because of inappropriate antibiotic usage. New antibiotics discoveries are not at par with the rapidly developing drug resistance. To tide over these circumstances, old antibiotics are now being revived researched and assigned new roles. Antibiotics, such as colistin, nitrofurantoin, polymyxin, etc., are used in nosocomial and critical care setting to tackle the multidrug-resistant bacteria. Even the use of old antibiotics should be pathogen centered and inappropriate use should be avoided. In this study, we are trying to throw some light on some of the old antibiotics which are currently in use and their efficacy. A lot of research is still required to optimize the drug dosing and prevention of side effects of these older antibiotics.
\end{abstract}

Keywords: Infections, Methicillin-resistant Staphylococcus aureus, Staphylococcus aureus.

Pediatric Infectious Disease (2020): 10.5005/jp-journals-10081-1242

The current medical practice involves use of a wide range of antibiotics for treating infections. The excessive and irrational use of antibiotics for mild illness has resulted in the emergence of a large number of resistant organisms especially which are resistant to a lot of commonly used antibiotics. Time and again practitioners are made aware of this emerging situation, and as days progress we will be devoid of all the available drugs as new antibiotic inventions are not keeping pace. ${ }^{1}$ This is a very scary and difficult scenario when it comes to the treatment of patients with nosocomial infections, patients with underlying immunodeficiency, and patients in critical care units. As we are left with very few new antibiotic options, the need of hour is to bring in the old and forgotten antibiotics in new forms and new roles.

This is a very important step at this juncture through which we can keep the new and expensive antibiotics as reserve and also prevent the development of drug resistance.

Older antibiotics went behind curtains mainly because of reasons, such as side effects, palatability, difficulty in route of administration, and advent of more effective agents. Before using it in clinical settings, older antibiotics safety and efficacy should be reevaluated to optimize therapy.

The most advanced revived antibiotic now commonly used in present clinical practice is colistin. Other antibiotics, such as nitrofurantoin, doxycycline, etc., are also currently used for various gram-positive and gram-negative infections which are resistant to new drugs.

\section{Polymyxins}

Polymyxins are group of antibiotics which came into use in the 1940s and went into recluse after few years because of the neurotoxic and nephrotoxic side effects. Among the group of five compounds, polymyxin B and polymyxin $E$ are used in clinical practice. ${ }^{2}$ They are bactericidal against gram-negative bacteria and not effective against majority of gram-positive bacteria and gram-negative cocci. Their use have resurfaced as they were shown to have in vitro activity against gramnegative bacteria, such as Klebsiella, Pseudomonas aeruginosa,
1,2Department of Pediatrics, Manipal Hospitals, Bengaluru, Karnataka, India

Corresponding Author: Anuradha Vinod, Department of Pediatrics, Manipal Hospitals, Bengaluru, Karnataka, India, Phone: +91 9632221282, e-mail: sreeram_anu_b@yahoo.com

How to cite this article: Vinod A, Shenoy B. Time to Fight the New with the Old-Old Antibiotics in New Role. Pediatr Inf Dis 2020;2(2):67-69.

Source of support: Nil

Conflict of interest: None

Enterobacteriaceae, etc., which were resistant to the most commonly used antibiotics.

\section{Colistin (Polymyxin E)}

Colistin also known as polymyxin E came into clinical use in the 1950 s and soon because of the neurotoxic and nephrotoxic side effects and the availability of other drugs with less side effects it went out of clinical usage. ${ }^{3}$ Recently, the focus on colistin has resurfaced because of its bactericidal effect on gram-negative bacteria majority of which are resistant to multiple antibiotics.

\section{Spectrum of Action}

Mainly bactericidal activity against major pathogenic gramnegative bacteria which are mainly involved in infections in immunocompromised, critical care settings, and patients with underlying chronic diseases like Enterobacteriaceae including extended spectrum beta-lactamase (ESBL) and carapenemase producing strains, such as Klebsiella pneumoniae, Acinetobacter baumannii, and Pseudomonas aeruginosa. Antibacterial spectrum also includes other bacteria, such as Escherichia coli, Shigella, Salmonella, Bordetella, Haemophilus influenzae, etc.

\section{Indications}

Number of multicentric studies have proven efficacy and guarded safety of colistin in children, infants, and neonates mainly for highly resistant gram-negative infections which are resistant 
to multiple drugs $s^{4,5}$ or in critical care settings where adequate clinical progress is not seen and proven culture sensitive infections. Initially, colistin found use mainly in post burn care patients ${ }^{6}$ and in those with cystic fibrosis. Main infections where it has found use include severe sepsis, ventilator-associated infections, meningitis, intra-abdominal infections, etc. It is always used as combination therapy even intrathecal use has been proved to be efficacious in newborns, etc. Role of colistin in critically ill children and nosocomial infection also proved it to be effective. ${ }^{7}$ Inhaled colistin has also found a place in gram-negative lung infections and mechanically ventilated patients.

\section{Polymyxin B}

\section{Spectrum of Action}

Polymyxin B is also used for resistant infections by Enterobacteriaceae, Pseudomonas, Klebsiella, and E. coli ${ }^{8}$ Mainly in nosocomial infections and infection in critical care settings. Multidrug-resistant gramnegative bacilli with high rates of carbapenem resistance is leading to increasing use of polymyxin $B$ as the only drug to combat against these infections in critically ill children. ${ }^{9}$

\section{Side Effects}

Nephrotoxicity and neurotoxicity are dose and duration dependent. Dosage adjustment need to be made in children with renal impairment. With proper titration of doses and monitoring of renal function, the side effects can be minimized. The nephrotoxicity induced by colistin was proved to be reversible according to certain studies and to a less extent neurotoxicity. ${ }^{5}$

\section{Nitrofurantoin}

Even though nitrofurantoin came into clinical practice in 1952, it was not used widely because of gastrointestinal side effects. After the macrocrystalline form came, side effects were less and safety profile improved.

\section{Spectrum of Activity}

Nitrofurantoin has a broad spectrum of antibacterial action ranging from gram-negative bacteria, such as E. coli, Klebsiella, Enterobacter, Shigella, Salmonella, etc., to gram-positive organisms, such as Staphylococcus aureus, group Streptococcus, etc. The most important feature is that $\mathrm{ESBL}$ producing $E$. coli is also sensitive to nitrofurantoin, so it is mainly indicated for the treatment of lower urinary tract infections ${ }^{10}$ due to the increase in ESBL producing $E$. coli emerging as a major pathogen in urinary tract infections. It also finds use in recurrent and treatment resistant infections which shows culture sensitivity. The disadvantage is that of the poor palatability almost making the drug non-compliant in children.

\section{Trimethoprim-SUlfamethoXazole}

Trimethoprim-sulfamethoxazole is a two-drug fixed combination antibiotic individually either of them are bacteriostatic together has a very broad spectrum of action against both gram-positive bacteria including some methicillin-resistant Staphylococcus aureus (MRSA) and gram-negative bacteria like Enterobacter. The drug combination was introduced in 1969. The drug is also effective against Toxoplasma gondii isospora and Pneumocystis jirovecii. Due to the two-drug combination resistance development to drug is less. The main advantage of the drug was good oral availability and cost effectiveness and disadvantage was the allergic reaction in susceptible patients.

\section{Spectrum of Action}

Due to its bactericidal activity against gram-negative bacteria, such as Enterobacter, E. coli, Klebsiella, we find its clinical use in urinary tract infections acute as well as recurrent, also indicated in prophylaxis in patients with underlying urinary tract pathologies. Based on the sensitivity pattern, it finds its use in respiratory infections like pneumonia, also in otitis media its previous role as a first-line drug in community-acquired pneumonia is fading due to gradual development of resistant organisms. In immunocompromised individuals, the drug has a major role due to its efficacy against $P$.jirovecii and isospora. The drug's role in typhoid and bacillary dysentery has also declined due to its resistance but it is one of the drugs of choice in brucellosis and nocardiosis. Emerging drug-resistant nosocomial infections have again shifted focus on trimethoprim-sulfamethoxazole. ${ }^{11}$ It is also likely to be effective in community-acquired MRSA and mainly used in skin and superficial infections.

The judicious use of trimethoprim-sulfamethoxazole will prevent overuse of costly and new antibiotics and preserve the drug for sensitive microbes preventing drug resistance.

\section{Clindamycin}

Clindamycin clinically came into use since 1962. Concerns about Clostridium difficile has limited its use. It is very useful against anaerobic infections. ${ }^{12}$

\section{Spectrum of Action}

Similar antibacterial spectrum like of macrolides. Effective against mainly gram-positive aerobic cocci like Staphylococcus even penicillinresistant Streptococcus pneumoniae ${ }^{13}$ and community-acquired methicillin-resistant $S$. aureus. Its effectiveness against anaerobes finds its usage in intra-abdominal, bone and joint infections, lung abscess, etc. Due to its inhibitory effect on bacterial toxin production, it finds role in necrotizing fasciitis, toxic shock syndrome (TSS), staphylococcal induced and also in streptococcal toxic shock either alone or as a combination therapy. Other advantages include good oral absorption and tissue distribution. Clindamycin is also used for resistant $P$. jirovecii and adjunctive in Plasmodium falciparum infections. Palatability is a disadvantage. Dosage adjustment required in hepatic and renal dysfunction. Major side effects are pseudomembranous colitis which is not dose or duration dependent, though, less reported in children. Judicious use of this drug will spare expensive broad spectrum and newer antibiotics.

\section{TEICOPLANIN}

Glycopeptide group of antibiotics came into clinical use since 1978. It requires more research in children.

\section{Spectrum of Activity}

Action mainly against gram-positive organisms, active against both methicillin-resistant and methicillin-sensitive S. aureus, S. pneumoniae, enterococci, ${ }^{14,15}$ Listeria, Corynebacterium, Clostridium, and anaerobic gram-positive cocci. Oral absorption is poor. Indicated for the prevention of bacterial endocarditis in the presence of penicillin allergies. Also clinically used in catheter-related infections, cerebrospinal fluid (CSF), shunt infection resistant, bone and soft tissue infection. Mainly reserved for the patients with neutropenia along with beta-lactam and aminoglycosides. Advantages when compared to vancomycin are single daily dosage, intramuscular administration, etc. Side effects are mainly rashes, drug-related fever, and dosage adjustment to be made in renal impairment. 


\section{Fosfomycin}

Fosfomycin has a broad spectrum of action and excellent safety profile with little or limited research in pediatrics. The drug is noted to have very good dissemination into tissues like bones.

\section{Spectrum of Activity}

Antibacterial spectrum include MRSA, multidrug-resistant gramnegative bacteremia (carbapenem-resistant Enterobacteriaceae), Staphylococcus epidermidis, Listeria, and resistant Pneumococcus. Fosfomycin is currently being researched for community-acquired lower urinary tract infections in pediatrics. ${ }^{16}$

\section{DOXYCYCLINE}

Doxycycline, a second generation tetracycline, came into clinical use since early 1970s. Uses in children were limited due to the side effects of discoloration of teeth. Recently, studies have proved against this and now doxycycline is recommended for shorter duration therapy even in children less than 8 years.

\section{Spectrum of Activity}

Doxycycline exhibits bacteriostatic effect on a large number of gram-positive and gram-negative bacteria parasites, etc., of which Brucella, Bartonella, Enterobacter, Gonococci, Vibrio cholerae, Bacillus anthrax, Listeria. Other organisms, such as Rickettsia, Mycoplasma, Chlamydia, etc., require special mention. It is considered as a firstline therapy in rickettsial illness in any age group. Also used in trachoma, psittacosis, relapsing fever, etc. Recently, doxycycline is used for macrolide-resistant Mycoplasma infections. In scenarios of resistant gram-negative infections, if culture sensitive then doxycycline is used. Use in cholera and prophylaxis for falciparum malaria also found to be effective. ${ }^{17}$ Due to its intracellular mechanism of activity recently approved safety profile and low cost, the demand of doxycycline in clinical practice has increased. ${ }^{18}$ Over use of this drug should be avoided and should be used only in recommended and culture proven conditions. Photosensitivity, C. difficile infection, and teeth discoloration on prolonged use and availability in the form of capsules are major disadvantages.

\section{TEMOCILLIN}

Temocillin, a semisynthetic penicillin derivative, came in 1980s did not see much clinical use due to its narrow antibacterial spectrum, mainly lack of action against gram-positive bacteria. The current rise of resistant gram-negative bacteria resulted in renewed interest in temocillin which has excellent beta-lactamase and carbapenemase stability.

\section{Spectrum of Activity}

As mentioned earlier, temocillin has a narrow spectrum of activity mainly against gram-negative bacilli, such as $E$. coli, Klebsiella, Enterobacter sp., etc. The clinical need of temocillin is its effectiveness against resistant Enterobacteriaceae ${ }^{4}$ ) and helps in reserving carbapenems. Temocillin proved to be effective and safe in the treatment of pyelonephritis often due to ampicillin-resistant strains in children according to a study by Verboven. ${ }^{19}$ Further studies in pediatrics is required now, as of limited studies the drug is mainly used in hepatic transplant recipients, resistant urinary infections, and cholangitis.

Many other old antibiotics, such as spectinomycin, minocycline, quinupristin-dalfopristin, fusidic acid, etc., are being extensively revived researched in adults.
Rational use of old antibiotics in clinical practice paves a new way in the treatment of multidrug-resistant pathogens which is very much pronounced in the critical care and nosocomial setting and slowly expanding into community. The old drugs also help in preserving the new and expensive drugs, so that we are better equipped in our fight against the microbes. Antibiotic stewardship is the current norm and must be followed in all clinical settings.

\section{References}

1. Cassir N, Rolain J-M, Brouqui. P. A new strategy to fight antimicrobial resistance: the revival of old antibiotics. Front Microbiol 2014;5:551. DOI: $10.3389 /$ fmicb.2014.00551.

2. Gupta S, Govil D, Kakar PN, et al. Colistin and polymyxin B: a re-emergence. Indian J Crit Care Med 2009;13(2):49-53. DOI: 10.4103/0972-5229.56048.

3. Celebi S, Hacimustafaoglu M, Koksal N. Colistimethate sodium therapy for multidrug-resistant isolates in pediatric patients. Pediatr Int 2010;52(3):410-414. DOI: 10.1111/j.1442-200X.2009.03015.x.

4. Iosifidis E. Colistin administration to pediatric and neonatal patients. Eur J Pediatr 2010;169(7):867-874. DOI: 10.1007/s00431-009-1137-3.

5. Jajoo M. Intravenous colistin administration in neonates. Pediatr Infect Dis J 2011;30(3):218-221. DOI: 10.1097/INF.0b013e3182064bfe.

6. Flagas ME. Systemic colistin use in children without cystic fibrosis: a systematic review of the literature. Int J Antimicrob Agents 2009;33:503.e1-50a3.e13.

7. Falagas ME. Intravenous colistimethate (colistin) use in critically ill children without cystic fibrosis. Pediatr Infect Dis J 2009;28(2):123-127. DOI: 10.1097/INF.0b013e31818a5dbd.

8. Tamma PD, Newland JG, Pannaraj PS, et al. The use of intravenous colistin among children in the United States: results from a multicenter, case series. Pediatr Infect Dis J 2013;32(1):17-22. DOI: 10.1097/INF.0b013e3182703790.

9. Siddiqui NR, Qamar FN, Jurair $\mathrm{H}$, et al. Multi-drug resistant gram negative infections and use of intravenous polymyxin B in critically ill children of developing country: retrospective cohort study. BMC Infect Dis 2014;14(1):626. DOI: 10.1186/s12879-014-0626-9.

10. Huttner A, Verhaegh EM, Harbarth $S$, et al. Nitrofurantoin revisited: a systematic review and meta-analysis of controlled trials, Mouton. J Antimicrob Chemother 2015;70(9):2456-2464. DOI: 10.1093/jac/dkv147.

11. Masters PA, O'Bryan TA, Zurlo J, et al. Trimethoprim-sulfamethoxazole revisited. Arch Intern Med 2003;163(4):402-410. DOI: 10.1001/ archinte.163.4.402.

12. Clindamycin: new look at an old drug. Infectious Diseases in Children, January 2005.

13. Doern GV. Antimicrobial resistance among clinical isolates of streptococcus pneumoniae in the United States during 19992000, including a comparison of resistance rates since 1994-1995. Antimicrob Agent Chemother 2001;45(6):1721-1729. DOI: 10.1128/ AAC.45.6.1721-1729.2001.

14. Van Bambeke F. Glycopeptides and glycodepsipeptides in clinical development: a comparative review of their antibacterial spectrum, pharmacokinetics and clinical efficacy. Curr Opin Investigat Drugs 2006;7(8):740-749.

15. Jeya M, Moon HJ, Lee KM, et al. Glycopeptide antibiotics and their novel semi-synthetic derivatives. Curr Pharm Biotechnol 2011;12(8):1194-1204. DOI: 10.2174/138920111796117382.

16. Baquero-Artigao F, del Rosal Rabes T. Fosfomycin in the pediatric setting: evidence and potential Indications. J Spanish Soc Chemothe 2019;32(Suppl 1):55-61.

17. Cross R, Ling C, Day NP, et al. Revisiting doxycycline in pregnancy and early childhood-time to rebuild its reputation? Expert opinion. Drug Saf 2016;15(3):367-382. DOI: 10.1517/14740338.2016.1133584.

18. Smith K, Leyden JJ. Safety of doxycycline and minocycline: a systematic review. Clin Ther 2005;27(9):1329-1342. DOI: 10.1016/ j.clinthera.2005.09.005.

19. Verboven $M$, Lauwers $S$, Pintens $H$. Temocillin in the treatment of pyelonephritis in children. Drugs Exp Clin Res 1987;113(3):171-173. 